\title{
ESSENTIAL NORM OF A VOLTERRA-TYPE INTEGRAL OPERATOR FROM HARDY SPACES TO SOME ANALYTIC FUNCTION SPACES
}

\author{
JIZHEN ZHOU AND XIANGLING ZHU
}

Communicated by Hermann Brunner

\begin{abstract}
In this paper, we obtain some estimates of essential norm of the Volterra-type integral operator $T_{g}$, where

$$
T_{g} f(z)=\int_{0}^{z} f(\zeta) g^{\prime}(\zeta) d \zeta
$$

from Hardy spaces to the BMOA space, Besov spaces, Bergman spaces and Bloch-type spaces.
\end{abstract}

1. Introduction. The space of all analytic functions on the unit disk $\mathbb{D}=\{z:|z|<1\}$ in the complex plane is denoted by $H(\mathbb{D})$. Let $0<p<\infty$. The Bergman space, denoted by $A^{p}$, is the space of all $f \in H(\mathbb{D})$ satisfying

$$
\|f\|_{A^{p}}^{p}=\int_{\mathbb{D}}|f(z)|^{p} d A(z)<\infty,
$$

where $d A$ is the normalized Lebesgue area measure in $\mathbb{D}$ such that $A(\mathbb{D})=1$. The Hardy space $H^{p}$ consists of all $f \in H(\mathbb{D})$ such that

$$
\|f\|_{H^{p}}^{p}=\sup _{0<r<1} \frac{1}{2 \pi} \int_{0}^{2 \pi}\left|f\left(r e^{i \theta}\right)\right|^{p} d \theta<\infty .
$$

2010 AMS Mathematics subject classification. Primary 30H10, 47 B38.

Keywords and phrases. Volterra-type integral operator, Hardy space, BMOA space.

The first author is supported by NSF of Anhui Province, grant Nos. 1308085QA12 and 1608085MA01 and the Fund of Anhui University of Science and Technology, grant No. QN201428. The second author is supported by NNSF of China, grant No. 11471143. The second author is the corresponding author.

Received by the editors on April 20, 2016. 
As usual, $H^{\infty}$ denotes the space of bounded analytic function. We say that an $f \in H(\mathbb{D})$ belongs to the BMOA space, if

$$
\|f\|_{*}^{2}=\sup _{I \subseteq \partial \mathbb{D}} \frac{1}{|I|} \int_{I}\left|f(\zeta)-f_{I}\right|^{2} \frac{d \zeta}{2 \pi}<\infty
$$

where $f_{I}=(1 /|I|) \int_{I} f(\zeta)(d \zeta / 2 \pi)$. It is well known that BMOA is a Banach space under the norm $\|f\|_{\text {BMOA }}=|f(0)|+\|f\|_{*}$. From [4], we have $\|f\|_{*}$ is comparable with $\sup _{w \in \mathbb{D}}\left\|f \circ \sigma_{w}-f(w)\right\|_{H^{2}}$, where $\sigma_{w}(z)=(w-z) /(1-\bar{w} z)$ is a Möbius transformation of $\mathbb{D}$. We say that an $f \in H(\mathbb{D})$ belongs to the VMOA space if

$$
\lim _{|w| \rightarrow 1}\left\|f \circ \sigma_{w}-f(w)\right\|_{H^{2}}=0 .
$$

For $\alpha>0$, we say that an $f \in H(\mathbb{D})$ belongs to Bloch-type space $\mathcal{B}^{\alpha}$ if

$$
\|f\|_{\mathcal{B}^{\alpha}}=\sup _{z \in \mathbb{D}}\left(1-|z|^{2}\right)^{\alpha}\left|f^{\prime}(z)\right|<\infty \text {. }
$$

An $f \in H(\mathbb{D})$ belongs to the little Bloch-type space $\mathcal{B}_{0}^{\alpha}$ if

$$
\lim _{|z| \rightarrow 1}\left(1-|z|^{2}\right)^{\alpha}\left|f^{\prime}(z)\right|=0 .
$$

Let $p>1$. The Besov space $\mathcal{B}_{p}$ consists of all $f \in H(\mathbb{D})$ such that

$$
\|f\|_{\mathcal{B}_{p}}^{p}=\int\left|f^{\prime}(z)\right|^{p}\left(1-|z|^{2}\right)^{p-2} d A(z)<\infty .
$$

Let $0<p, s<\infty,-2<q<\infty$. An $f \in H(\mathbb{D})$ is said to belong to the space $F(p, q, s)$ if, see [19],

$$
\|f\|_{p, q, s}^{p}=\sup _{a \in \mathbb{D}} \int_{\mathbb{D}}\left|f^{\prime}(z)\right|^{p}\left(1-|z|^{2}\right)^{q}\left(1-\left|\sigma_{a}(z)\right|^{2}\right)^{s} d A(z)<\infty .
$$

An $f \in H(\mathbb{D})$ belongs to the space $F_{0}(p, q, s)$ if

$$
\lim _{|a| \rightarrow 1} \int_{\mathbb{D}}\left|f^{\prime}(z)\right|^{p}\left(1-|z|^{2}\right)^{q}\left(1-\left|\sigma_{a}(z)\right|^{2}\right)^{s} d A(z)=0 .
$$

The $F(p, q, s)$ space becomes a Banach space with the norm $\|f\|_{F(p, q, s)}$ $=|f(0)|+\|f\|_{p, q, s} . F(p, q, s)$ is called general function space since it can get many function spaces by taking special parameters of $p, q, s$. For example, $F(2,1,0)=H^{2}, F(p, p, 0)=A^{p}, F(2,0,1)=$ BMOA 
and $F(p, q, s)=\mathcal{B}^{(q+2) / p}$ for $s>1$. We denote $F(p, p \alpha-2,1)$ and $F_{0}(p, p \alpha-2,1)$ by $\mathrm{BMOA}_{p}^{\alpha}$ and $\mathrm{BMOA}_{p, 0}^{\alpha}$, respectively.

Let $X$ and $Y$ be two Banach spaces. The essential norm of a bounded linear operator $T$ between $X$ and $Y$ is defined as follows.

$$
\|T\|_{e}^{X \rightarrow Y}=\inf \left\{\|T-K\|^{X \rightarrow Y}: K \text { is compact }\right\},
$$

where $\|\cdot\|^{X \rightarrow Y}$ is the operator norm. It is easy to see that $\|T\|_{e}^{X \rightarrow Y}=0$ if and only if $T$ is compact. For two Banach spaces $X$ and $Y$ with $Y \subset X$, if $f \in X$, then the distance of $f$ to the space $Y$ is defined by

$$
\operatorname{dist}_{X}(f, Y)=\inf _{h \in Y}\|f-h\|_{X} .
$$

For any $g \in H(\mathbb{D})$, the Volterra-type integral operator $T_{g}$ is defined as follows:

$$
T_{g} f(z)=\int_{0}^{z} f(\zeta) g^{\prime}(\zeta) d \zeta, \quad z \in \mathbb{D}, f \in H(\mathbb{D}) .
$$

The operator $T_{g}$ was introduced by Pommerenke [14], and he proved that $T_{g}$ is bounded on the Hardy space $H^{2}$ if and only if $g$ belongs to BMOA. In [1], Aleman and Siskakis showed that Pommerenke's boundedness characterization is valid on each $H^{p}$ for $1 \leq p<\infty$ and that $T_{g}$ is compact on $H^{p}$ if and only if $g \in \mathrm{VMOA}$. The boundedness and compactness of the operator $T_{g}$ on some holomorphic spaces, as well as its extension on the unit ball, were investigated, for example, $[1,2,5,6,7,8,9,10,11,12,13,14,15,16,17,20,21,22]$ (and related references therein).

Recently, many researchers have also been interested in the study of the essential norm of various operators. Laitila, Miihkinen and Nieminen [7] studied the essential norm of the operator $T_{g}$ on the Hardy space. Liu, Lou and Xiong [13] studied the essential norm of the operator $T_{g}$ on the Bloch space and some other spaces. Zhuo and Ye studied the essential norm of the operator $T_{g}$ from Morrey spaces to the Bloch space [22].

Zhao [20] obtained some characterizations of the operator $T_{g}$ from Hardy spaces to some other analytic function spaces. Therefore, it is also interesting to study the essential norm of the operator $T_{g}$ on these spaces. The main purpose of this paper is to obtain some estimates 
for the essential norm of the operator $T_{g}$ from Hardy spaces $H^{p}$ to the BMOA space, Besov spaces, Bergman spaces and Bloch-type spaces.

Throughout the paper, we say that $A \lesssim B$ if there exists a constant $C$ such that $A \leq C B$. The symbol $A \approx B$ means that $A \lesssim B \lesssim A$.

2. Essential norms of $T_{g}$. In this section, we will state our main results and proofs. For this purpose, we need some useful lemmas as follows.

Lemma 2.1 ([20]). Let $g \in H(\mathbb{D}), p \geq 1, \alpha>0$ and $\alpha-1 / p>0$. Then the following statements hold.

(i) $T_{g}: H^{p} \rightarrow \mathrm{BMOA}(p>1)$ is bounded if and only if $g \in \mathcal{B}^{1-1 / p}$, $T_{g}: H^{p} \rightarrow \mathrm{BMOA}$ is compact if and only if $g \in \mathcal{B}_{0}^{1-1 / p}$.

(ii) $T_{g}: H^{p} \rightarrow \mathcal{B}^{\alpha}$ is bounded if and only if $g \in \mathcal{B}^{\alpha-1 / p}, T_{g}: H^{p} \rightarrow \mathcal{B}^{\alpha}$ is compact if and only if $g \in \mathcal{B}_{0}^{\alpha-1 / p}$.

(iii) $T_{g}: H^{p} \rightarrow B_{p}(p>1)$ is bounded if and only if $g \in \mathrm{BMOA}_{p}^{1-1 / p}$, $T_{g}: H^{p} \rightarrow B_{p}$ is compact if and only if $g \in \mathrm{BMOA}_{p, 0}^{1-1 / p}$.

(iv) $T_{g}: H^{p} \rightarrow A^{p}$ is bounded if and only if $g \in \mathrm{BMOA}_{p}^{1+1 / p}$, $T_{g}: H^{p} \rightarrow A^{p}$ is compact if and only if $g \in \mathrm{BMOA}_{p, 0}^{1+1 / p}$.

Remark 2.2. When $p=1$, from [20, Theorem 11], we see that $T_{g}$ : $H^{1} \rightarrow \mathrm{BMOA}$ is bounded if and only if $g^{\prime} \in H^{\infty} . T_{g}: H^{1} \rightarrow \mathrm{BMOA}$ is compact if and only if $g$ is a constant.

The next lemma can be proved similarly as [18]. For the completeness of this paper, we include the proof here.

Lemma 2.3. If $\alpha>0$ and $g \in \mathcal{B}^{\alpha}$, then

$$
\operatorname{dist}_{\mathcal{B}^{\alpha}}\left(g, \mathcal{B}_{0}^{\alpha}\right) \approx \limsup _{|z| \rightarrow 1}\left(1-|z|^{2}\right)^{\alpha}\left|g^{\prime}(z)\right| \text {. }
$$

Proof. Denote by $f_{r}(z)=f(r z)$ for $0<r<1$. For any given $g \in \mathcal{B}^{\alpha}$, then $g_{r} \in \mathcal{B}_{0}^{\alpha}$ and $\left\|g_{r}\right\|_{\mathcal{B}^{\alpha}} \lesssim\|g\|_{\mathcal{B}^{\alpha}}$. For any given $\delta \in(0,1)$, it is easy to see that

$$
\lim _{r \rightarrow 1} \sup _{|z| \leq \delta}\left(1-|z|^{2}\right)\left|g^{\prime}(z)-g_{r}^{\prime}(z)\right|=0
$$


which implies

$$
\begin{aligned}
\operatorname{dist}_{\mathcal{B}^{\alpha}}\left(g, \mathcal{B}_{0}^{\alpha}\right)= & \inf _{f \in \mathcal{B}_{0}^{\alpha}}\|g-f\|_{\mathcal{B}^{\alpha}} \leq \lim _{r \rightarrow 1}\left\|g-g_{r}\right\|_{\mathcal{B}^{\alpha}} \\
= & \lim _{r \rightarrow 1} \sup _{|z|>\delta}\left(1-|z|^{2}\right)^{\alpha}\left|g^{\prime}(z)-r g^{\prime}(r z)\right| \\
& +\lim _{r \rightarrow 1} \sup _{|z| \leq \delta}\left(1-|z|^{2}\right)\left|g^{\prime}(z)-g_{r}^{\prime}(z)\right| \\
\leq & \sup _{|z|>\delta}\left(1-|z|^{2}\right)^{\alpha}\left|g^{\prime}(z)\right|+\lim _{r \rightarrow 1} \sup _{|z|>\delta}\left(1-|z|^{2}\right)^{\alpha}\left|r g^{\prime}(r z)\right| .
\end{aligned}
$$

Since $\delta$ is arbitrary, we have $\operatorname{dist}_{\mathcal{B}^{\alpha}}\left(g, \mathcal{B}_{0}^{\alpha}\right) \lesssim \lim \sup _{|z| \rightarrow 1}\left(1-|z|^{2}\right)^{\alpha}\left|g^{\prime}(z)\right|$.

On the other hand, for any $f \in \mathcal{B}_{0}^{\alpha}$,

$$
\begin{aligned}
\|g-f\|_{\mathcal{B}^{\alpha}} & \geq \limsup _{|z| \rightarrow 1}\left(1-|z|^{2}\right)\left|g^{\prime}(z)-f^{\prime}(z)\right| \\
& =\limsup _{|z| \rightarrow 1}\left(1-|z|^{2}\right)^{\alpha}\left|g^{\prime}(z)\right| .
\end{aligned}
$$

This yields

$$
\operatorname{dist}_{\mathcal{B}^{\alpha}}\left(g, \mathcal{B}_{0}^{\alpha}\right)=\inf _{f \in \mathcal{B}_{0}^{\alpha}}\|g-f\|_{\mathcal{B}^{\alpha}} \geq \limsup _{|z| \rightarrow 1}\left(1-|z|^{2}\right)^{\alpha}\left|g^{\prime}(z)\right|,
$$

as desired. The proof is complete.

Lemma 2.4. ([7, Lemma 3]). Suppose $g \in$ BMOA. Then

$$
\begin{aligned}
\operatorname{dist}_{\mathrm{BMOA}}(g, \mathrm{VMOA}) & \approx \limsup _{r \rightarrow 1}\left\|g-g_{r}\right\|_{\mathrm{BMOA}} \\
& \approx \limsup _{|a| \rightarrow 1}\left\|g \circ \sigma_{a}-g(a)\right\|_{H^{2}} .
\end{aligned}
$$

Here $g_{r}(z)=g(r z)$ with $0<r<1$.

Lemma 2.5. Let $p>0$ and $\alpha>0$. If $g \in \mathrm{BMOA}_{p}^{\alpha}$, then

$$
\begin{aligned}
& \operatorname{dist}_{\mathrm{BMOA}_{\mathrm{p}}^{\alpha}}\left(g, \operatorname{BMOA}_{\mathrm{p}, 0}^{\alpha}\right) \\
& \qquad \limsup _{|a| \rightarrow 1} \int_{\mathbb{D}}\left|g^{\prime}(z)\right|^{2}\left(1-|z|^{2}\right)^{p \alpha-2}\left(1-\left|\sigma_{a}(z)\right|^{2}\right) d A(z) .
\end{aligned}
$$

Proof. Denote by $f_{r}(z)=f(r z)$ for $0<r<1$. For any given $g \in \mathrm{BMOA}_{p}^{\alpha}$, then $g_{r} \in \mathrm{BMOA}_{p, 0}^{\alpha}$ and $\left\|g_{r}\right\|_{\mathrm{BMOA}_{\mathrm{p}}^{\alpha}} \lesssim\|g\|_{\mathrm{BMOA}_{\mathrm{p}}^{\alpha}}$. Let 
$\delta \in(0,1)$. We choose $a \in(0, \delta)$. Then $\sigma_{a}(z)$ lies in a compact subset of $\mathbb{D}$. So

$$
\limsup _{r \rightarrow 1}\left|g_{z \in \mathbb{D}}\left(\sigma_{a}(z)\right)-r g^{\prime}\left(r \sigma_{a}(z)\right)\right|=0 .
$$

Making a change of variables, we have

$$
\begin{aligned}
& \lim _{r \rightarrow 1} \sup _{|a| \leq \delta} \int_{\mathbb{D}}\left|g^{\prime}(z)-g_{r}^{\prime}(z)\right|^{p}\left(1-|z|^{2}\right)^{p \alpha-2}\left(1-\left|\sigma_{a}(z)\right|^{2}\right) d A(z) \\
& \quad=\lim _{r \rightarrow 1} \sup _{|a| \leq \delta} \int_{\mathbb{D}}\left|g^{\prime}\left(\sigma_{a}(z)\right)-g_{r}^{\prime}\left(\sigma_{a}(z)\right)\right|^{p}\left(1-|z|^{2}\right)^{p \alpha-1}\left|\sigma_{a}^{\prime}(z)\right|^{p \alpha} d A(z) \\
& \quad=\lim _{r \rightarrow 1} \sup _{|a| \leq \delta} \sup _{z \in \mathbb{D}}\left|g^{\prime}\left(\sigma_{a}(z)\right)-g_{r}^{\prime}\left(\sigma_{a}(z)\right)\right|^{p} \int_{\mathbb{D}}\left(1-|z|^{2}\right)^{p \alpha-1}\left|\sigma_{a}^{\prime}(z)\right|^{p \alpha} d A(z)=0 .
\end{aligned}
$$

By the definition of distance, we obtain

$$
\begin{aligned}
\operatorname{dist}_{\mathrm{BMOA}_{\mathrm{p}}^{\alpha}}\left(g, \mathrm{BMOA}_{p, 0}^{\alpha}\right)=\inf _{f \in \mathrm{BMOA}_{\mathrm{p}, 0}^{\alpha}}\|g-f\|_{\mathrm{BMOA}_{\mathrm{p}}^{\alpha}} \\
\leq \lim _{r \rightarrow 1}\left\|g-g_{r}\right\|_{\mathrm{BMOA}_{\mathrm{p}}^{\alpha}} \\
=\lim _{r \rightarrow 1} \sup _{|a|>\delta} \int_{\mathbb{D}}\left|g^{\prime}(z)-g_{r}^{\prime}(z)\right|^{p}\left(1-|z|^{2}\right)^{p \alpha-2}\left(1-\left|\sigma_{a}(z)\right|^{2}\right) d A(z) \\
\quad+\lim _{r \rightarrow 1} \sup _{|a| \leq \delta} \int_{\mathbb{D}}\left|g^{\prime}(z)-g_{r}^{\prime}(z)\right|^{p}\left(1-|z|^{2}\right)^{p \alpha-2}\left(1-\left|\sigma_{a}(z)\right|^{2}\right) d A(z) \\
\lesssim \sup _{|a|>\delta} \int_{\mathbb{D}}\left|g^{\prime}(z)\right|^{p}\left(1-|z|^{2}\right)^{p \alpha-2}\left(1-\left|\sigma_{a}(z)\right|^{2}\right) d A(z) \\
\quad+\lim _{r \rightarrow 1} \sup _{|a|>\delta} \int_{\mathbb{D}}\left|g_{r}^{\prime}(z)\right|^{p}\left(1-|z|^{2}\right)^{p \alpha-2}\left(1-\left|\sigma_{a}(z)\right|^{2}\right) d A(z) .
\end{aligned}
$$

Denote by $\psi_{r, a}(z)=\sigma_{r a} \circ r \sigma_{a}(z)$. Then $\psi_{r, a}$ is an analytic self-map of $\mathbb{D}$ and $\psi_{r, a}(0)=0$. Making a change of variable of $z=\sigma_{a}(z)$ and applying Littlewood's subordination theorem (see [3, Theorem 1.7]), we have

$$
\begin{aligned}
& \int_{\mathbb{D}}\left|g_{r}^{\prime}(z)\right|^{p}\left(1-|z|^{2}\right)^{p \alpha-2}\left(1-\left|\sigma_{a}(z)\right|^{2}\right) d A(z) \\
& \quad=\int_{\mathbb{D}}\left|g_{r}^{\prime}\left(\sigma_{a}(z)\right)\right|^{p}\left(1-\left|\sigma_{a}(z)\right|^{2}\right)^{p \alpha}\left(1-|z|^{2}\right)^{-1} d A(z) \\
& \quad \leq \int_{\mathbb{D}}\left|g^{\prime} \circ \sigma_{r a} \circ \psi_{r, a}(z)\right|^{p}\left(1-\left|\sigma_{r a} \circ \psi_{r, a}(z)\right|^{2}\right)^{p \alpha}\left(1-|z|^{2}\right)^{-1} d A(z)
\end{aligned}
$$




$$
\begin{aligned}
& \leq \int_{\mathbb{D}}\left|g^{\prime} \circ \sigma_{r a} \circ \psi_{r, a}(z)\right|^{p}\left(1-\left|\sigma_{r a} \circ \psi_{r, a}(z)\right|^{2}\right)^{p \alpha}\left(1-|z|^{2}\right)^{-1} d A(z) \\
& \leq \int_{\mathbb{D}}\left|g^{\prime} \circ \sigma_{r a}(z)\right|^{p}\left(1-\left|\sigma_{r a}(z)\right|^{2}\right)^{p \alpha}\left(1-|z|^{2}\right)^{-1} d A(z) \\
& \leq \int_{\mathbb{D}}\left|g^{\prime}(z)\right|^{p}\left(1-|z|^{2}\right)^{p \alpha-2}\left(1-\left|\sigma_{r a}(z)\right|^{2}\right) d A(z) .
\end{aligned}
$$

Since $\delta$ is arbitrary, we get

$$
\begin{aligned}
& \operatorname{dist}_{\mathrm{BMOA}_{\mathrm{p}}^{\alpha}}\left(g, \mathrm{BMOA}_{p, 0}^{\alpha}\right) \\
& \quad \lesssim \limsup _{|a| \rightarrow 1} \int_{\mathbb{D}}\left|g^{\prime}(z)\right|^{p}\left(1-|z|^{2}\right)^{p \alpha-2}\left(1-\left|\sigma_{a}(z)\right|^{2}\right) d A(z) .
\end{aligned}
$$

On the other hand, for any $f \in \mathrm{BMOA}_{p}^{\alpha}$,

$$
\begin{aligned}
\operatorname{dist}_{\mathrm{BMOA}_{\mathrm{p}}^{\alpha}} & \left(g, \mathrm{BMOA}_{p, 0}^{\alpha}\right)=\inf _{f \in \mathrm{BMOA}_{p, 0}^{\alpha}}\|g-f\|_{\mathrm{BMOA}_{\mathrm{p}}^{\alpha}} \\
& \gtrsim \limsup _{|a| \rightarrow 1} \int_{\mathbb{D}}\left|g^{\prime}(z)\right|^{p}\left(1-|z|^{2}\right)^{p \alpha-2}\left(1-\left|\sigma_{a}(z)\right|^{2}\right) d A(z),
\end{aligned}
$$

which, together with equation (2.1), implies the desired result. The proof is complete.

Theorem 2.6. Let $g \in H(\mathbb{D})$ and $p>1$. Suppose that $T_{g}: H^{p} \rightarrow$ BMOA is bounded. Then

$$
\left\|T_{g}\right\|_{e}^{H^{p} \rightarrow \mathrm{BMOA}} \approx \limsup _{|z| \rightarrow 1}\left(1-|z|^{2}\right)^{1-1 / p}\left|g^{\prime}(z)\right| .
$$

Proof. First, we prove the upper estimate for the essential norm of $T_{g}$. For each $h \in \mathcal{B}_{0}^{1-1 / p}$, the operator $T_{h}: H^{p} \rightarrow$ BMOA is compact by Lemma 2.1. Moreover, by the linearity of $T_{g}$ respect to $g$, we have $\left\|T_{g}\right\|_{e}^{H^{p} \rightarrow \mathrm{BMOA}} \leq\left\|T_{g}-T_{h}\right\|^{H^{p} \rightarrow \mathrm{BMOA}}=\left\|T_{g-h}\right\|^{H^{p} \rightarrow \mathrm{BMOA}} \lesssim\|g-h\|_{\mathcal{B}^{1-1 / p}}$.

Hence,

$$
\begin{aligned}
\left\|T_{g}\right\|_{e}^{H^{p} \rightarrow \mathrm{BMOA}} & \lesssim \inf _{h \in \mathcal{B}_{0}^{1-1 / p}}\|g-h\|_{\mathcal{B}^{1-1 / p}}=\operatorname{dist}_{\mathcal{B}^{1-1 / p}}\left(g, \mathcal{B}_{0}^{1-1 / p}\right) \\
& \approx \limsup _{|z| \rightarrow 1}\left(1-|z|^{2}\right)^{1-1 / p}\left|g^{\prime}(z)\right|
\end{aligned}
$$


For any $a \in \mathbb{D}$, we define

$$
f_{a}(z)=\left[\frac{1-|a|^{2}}{(1-\bar{a} z)^{2}}\right]^{1 / p} .
$$

Taking $z=\mathrm{re}^{i \theta}$ and the Poisson integral formula gives the following:

$$
\begin{aligned}
\left\|f_{a}\right\|_{H^{p}} & =\sup _{0<r<1}\left(\frac{1}{2 \pi} \int_{0}^{2 \pi}\left|f_{a}\left(\mathrm{re}^{i \theta}\right)\right|^{p} d \theta\right)^{1 / p} \\
& =\sup _{0<r<1}\left(\frac{1}{2 \pi} \int_{0}^{2 \pi} \frac{1-|a|^{2}}{\left|1-\bar{a} \mathrm{re}^{i \theta}\right|^{2}} d \theta\right)^{1 / p} \\
& =\sup _{0<r<1}\left(\frac{1-|a|^{2}}{1-|a r|^{2}}\right)^{1 / p}=1 .
\end{aligned}
$$

In the meantime, we have $\left|f_{a}(a)\right|\left(1-|a|^{2}\right)^{1 / p}=1$. Since $f_{a} \rightarrow 0$ weakly in $H^{p}$ as $|a| \rightarrow 1$, we have $\left\|K f_{a}\right\|_{\mathrm{BMOA}} \rightarrow 0$ as $|a| \rightarrow 1$ for any compact operator $K: H^{p} \rightarrow$ BMOA. Moreover,

$\left\|T_{g}-K\right\|^{H^{p} \rightarrow \mathrm{BMOA}} \geq\left\|\left(T_{g}-K\right) f_{a}\right\|_{\mathrm{BMOA}} \geq\left\|T_{g} f_{a}\right\|_{\mathrm{BMOA}}-\left\|K f_{a}\right\|_{\mathrm{BMOA}}$.

Therefore,

$\left\|T_{g}-K\right\|^{H^{p} \rightarrow \mathrm{BMOA}} \geq \limsup _{|a| \rightarrow 1}\left\|\left(T_{g}-K\right) f_{a}\right\|_{\mathrm{BMOA}} \geq \limsup _{|a| \rightarrow 1}\left\|T_{g} f_{a}\right\|_{\mathrm{BMOA}}$,

which implies that

$$
\left\|T_{g}\right\|_{e}^{H^{p} \rightarrow \mathrm{BMOA}} \geq \limsup _{|a| \rightarrow 1}\left\|T_{g} f_{a}\right\|_{\mathrm{BMOA}} .
$$

In addition, by [19, Lemma 2.9], we have

$$
\begin{aligned}
\left\|T_{g} f_{a}\right\|_{\mathrm{BMOA}} & =\sqrt{\sup _{b \in \mathbb{D}} \int_{\mathbb{D}}\left|\left(T_{g} f_{a}\right)^{\prime}(z)\right|^{2}\left(1-\left|\sigma_{b}(z)\right|\right) d A(z)} \\
& \geq \sqrt{\int_{\mathbb{D}}\left|\left(T_{g} f_{a}\right)^{\prime}(z)\right|^{2}\left(1-\left|\sigma_{a}(z)\right|\right) d A(z)} \\
& =\sqrt{\int_{\mathbb{D}}\left|g^{\prime}(z)\right|^{2}\left(1-|z|^{2}\right)^{-2 / p}\left(1-\left|\sigma_{a}(z)\right|^{2}\right)^{1+2 / p} d A(z)} \\
& \gtrsim\left(1-|a|^{2}\right)^{1-1 / p}\left|g^{\prime}(a)\right| .
\end{aligned}
$$


Therefore,

$$
\left\|T_{g}\right\|_{e}^{H^{p} \rightarrow \mathrm{BMOA}} \geq \limsup _{|a| \rightarrow 1}\left\|T_{g} f_{a}\right\|_{\mathrm{BMOA}} \gtrsim \limsup _{|a| \rightarrow 1}\left(1-|a|^{2}\right)^{1-1 / p}\left|g^{\prime}(a)\right| .
$$

Then inequality (2.2) combined with the last inequality gives the desired result. The proof is complete.

Remark 2.7. When $p=1$, from Remark 2.2 and the definition of the essential norm operator we see that $\left\|T_{g}\right\|_{e}^{H^{1} \rightarrow \mathrm{BMOA}}=0$.

Theorem 2.8. Let $g \in H(\mathbb{D}), p \geq 1$ and $\alpha>1 / p$. Suppose that $T_{g}: H^{p} \rightarrow \mathcal{B}^{\alpha}$ is bounded. Then

$$
\left\|T_{g}\right\|_{e}^{H^{p} \rightarrow \mathcal{B}^{\alpha}} \approx \limsup _{|z| \rightarrow 1}\left(1-|z|^{2}\right)^{\alpha-1 / p}\left|g^{\prime}(z)\right| .
$$

Proof. The upper estimate for the essential norm of $T_{g}$ is similar to the proof of Theorem 2.6. We omit the details of the proof.

Now we only give the proof for the lower estimate. For any $a \in \mathbb{D}$, we choose the test function $f_{a}$ which is defined in equation (2.3). Since $f_{a} \rightarrow 0$ weakly in $H^{p}$ as $|a| \rightarrow 1$, we have

$$
\left\|K f_{a}\right\|_{\mathcal{B}^{\alpha}} \longrightarrow 0 \quad \text { as }|a| \rightarrow 1
$$

for any compact operator $K: H^{p} \rightarrow \mathcal{B}^{\alpha}$. Thus,

$$
\begin{aligned}
\left\|T_{g}-K\right\|^{H^{p} \rightarrow \mathcal{B}^{\alpha}} & \geq \limsup _{|a| \rightarrow 1}\left\|\left(T_{g}-K\right) f_{a}\right\|_{\mathcal{B}^{\alpha}} \\
& \geq \limsup _{|a| \rightarrow 1}\left\|T_{g} f_{a}\right\|_{\mathcal{B}^{\alpha}}-\limsup _{|a| \rightarrow 1}\left\|K f_{a}\right\|_{\mathcal{B}^{\alpha}} .
\end{aligned}
$$

Note that

$$
\begin{aligned}
\left\|T_{g} f_{a}\right\|_{\mathcal{B}^{\alpha}} & =\sup _{z \in \mathbb{D}}\left|\left(T_{g} f_{a}\right)^{\prime}(z)\right|\left(1-|z|^{2}\right)^{\alpha} \geq\left|\left(T_{g} f_{a}\right)^{\prime}(z)\right|\left(1-|a|^{2}\right)^{\alpha} \\
& =\left|g^{\prime}(a)\right|\left(1-|a|^{2}\right)^{\alpha-1 / p}
\end{aligned}
$$

The last inequality gives

$$
\left\|T_{g}\right\|_{e}^{H^{p} \rightarrow \mathcal{B}^{\alpha}} \geq \limsup _{|a| \rightarrow 1}\left\|T_{g} f_{a}\right\|_{\mathcal{B}^{\alpha}} \gtrsim \limsup _{|a| \rightarrow 1}\left(1-|a|^{2}\right)^{\alpha-1 / p}\left|g^{\prime}(a)\right| .
$$

The proof is complete. 
Theorem 2.9. Let $g \in H(\mathbb{D})$ and $p>1$. Suppose that $T_{g}: H^{p} \rightarrow \mathcal{B}_{p}$ is bounded. Then,

$$
\left\|T_{g}\right\|_{e}^{H^{p} \rightarrow \mathcal{B}_{p}} \approx \limsup _{|a| \rightarrow 1} \int_{\mathbb{D}}\left|g^{\prime}(z)\right|^{p}\left(1-|z|^{2}\right)^{p-3}\left(1-\left|\sigma_{a}(z)\right|^{2}\right) d A(z) .
$$

Proof. First, we consider the upper estimate for the essential norm of $T_{g}$. Indeed, for each $h \in \mathrm{BMOA}_{p, 0}^{1-1 / p}$, the operator $T_{h}$ is compact from $H^{p}$ to $\mathcal{B}_{p}$ by Lemma 2.1. Moreover,

$\left\|T_{g}\right\|_{e}^{H^{p} \rightarrow \mathcal{B}_{p}} \leq\left\|T_{g}-T_{h}\right\|^{H^{p} \rightarrow \mathcal{B}_{p}}=\left\|T_{g-h}\right\|^{H^{p} \rightarrow \mathcal{B}_{p}} \lesssim\|g-h\|_{\mathrm{BMOA}_{p}^{1-1 / p} .}$

Hence, by Lemma 2.4 and inequality (2.4) we have

$$
\begin{aligned}
\left\|T_{g}\right\|_{e}^{H^{p} \rightarrow \mathcal{B}_{p}} & \lesssim \inf _{h \in \mathrm{BMOA}_{p, 0}^{1-1 / p}}\|g-h\|_{\mathrm{BMOA}_{\mathrm{p}}^{1-1 / \mathrm{p}}} \\
& =\operatorname{dist}_{\mathrm{BMOA}_{\mathrm{p}}^{1-1 / \mathrm{p}}\left(g, \mathrm{BMOA}_{\mathrm{p}, 0}^{1-1 / \mathrm{p}}\right)} \\
& \approx \limsup _{|a| \rightarrow 1} \int_{\mathbb{D}}\left|g^{\prime}(z)\right|^{p}\left(1-|z|^{2}\right)^{p-3}\left(1-\left|\sigma_{a}(z)\right|^{2}\right) d A(z) .
\end{aligned}
$$

Let $f_{a}$ be defined as in equation (2.3). Since $f_{a} \rightarrow 0$ weakly in $H^{p}$ as $|a| \rightarrow 1$, we have $\left\|K f_{a}\right\|_{\mathcal{B}_{p}} \rightarrow 0$ as $|a| \rightarrow 1$ for any compact operator $K: H^{p} \rightarrow \mathcal{B}_{p}$. In addition,

$$
\left\|T_{g}-K\right\|^{H^{p} \rightarrow \mathcal{B}_{p}} \geq\left\|\left(T_{g}-K\right) f_{a}\right\|_{\mathcal{B}_{p}} \geq\left\|T_{g} f_{a}\right\|_{\mathcal{B}_{p}}-\left\|K f_{a}\right\|_{\mathcal{B}_{p}}
$$

we have

$$
\left\|T_{g}-K\right\|^{H^{p} \rightarrow \mathcal{B}_{p}} \geq \limsup _{|a| \rightarrow 1}\left\|\left(T_{g}-K\right) f_{a}\right\|_{\mathcal{B}_{p}} \geq \limsup _{|a| \rightarrow 1}\left\|T_{g} f_{a}\right\|_{\mathcal{B}_{p}}
$$

Since

$$
\begin{aligned}
\left\|T_{g} f_{a}\right\|_{\mathcal{B}_{p}} & =\int_{\mathbb{D}}\left|\left(T_{g} f_{a}\right)^{\prime}(z)\right|^{p}\left(1-|z|^{2}\right)^{p-2} d A(z) \\
& =\int_{\mathbb{D}}\left|g^{\prime}(z)\right|^{p}\left(1-|z|^{2}\right)^{p-3}\left(1-\left|\sigma_{a}(z)\right|\right) d A(z),
\end{aligned}
$$


we get

(2.6) $\left\|T_{g}\right\|_{e}^{H^{p} \rightarrow \mathcal{B}_{p}} \gtrsim \limsup _{|a| \rightarrow 1} \int_{\mathbb{D}}\left|g^{\prime}(z)\right|^{p}\left(1-|z|^{2}\right)^{p-3}\left(1-\left|\sigma_{a}(z)\right|^{2}\right) d A(z)$.

Then, inequality (2.5) combined with inequality (2.6) gives the desired result. The proof is complete.

Remark 2.10. When $p=1$, the definition of Besov space is completely different than the case of $p>1$. The analytic Besov space $\mathcal{B}_{1}$ is defined to be the set of all $f \in H(\mathbb{D})$ which can be written as

$$
f(z)=\sum_{n=1}^{\infty} a_{n} \sigma_{\lambda_{n}}(z)
$$

for $\left\{a_{n}\right\}$ in $l^{1}$ and $\lambda_{n} \in \mathbb{D}$. The norm of $\mathcal{B}_{1}$ is defined by

$$
\|f\|_{\mathcal{B}_{1}}=\inf \left\{\sum_{n=1}^{\infty}\left|a_{n}\right|: f(z)=\sum_{n=1}^{\infty} a_{n} \sigma_{\lambda_{n}}(z)\right\}
$$

It is obvious that $\mathcal{B}_{1} \subset H^{\infty} \subset$ BMOA. From Remarks 2.2 and 2.7 we see that $T_{g}: H^{1} \rightarrow \mathcal{B}_{1}$ is compact if and only if $g$ is a constant. Moreover, $\left\|T_{g}\right\|_{e}^{H^{1} \rightarrow \mathrm{BMOA}}=0$.

Similarly to the proof of Theorem 2.9, we immediately get the following result. We omit the proof here.

Theorem 2.11. Let $g \in H(\mathbb{D})$ and $p \geq 1$. Suppose that $T_{g}: H^{p} \rightarrow A^{p}$ is bounded. Then

$$
\left\|T_{g}\right\|_{e}^{H^{p} \rightarrow A^{p}} \approx \limsup _{|a| \rightarrow 1} \int_{\mathbb{D}}\left|g^{\prime}(z)\right|^{p}\left(1-|z|^{2}\right)^{p-1}\left(1-\left|\sigma_{a}(z)\right|^{2}\right) d A(z) .
$$




\section{REFERENCES}

1. A. Aleman and A. Siskakis, An integral operator on $H^{p}$, Complex Var. 28 (1995), 140-158.

2. Integral operators on Bergman spaces, Indiana Univ. Math. J. 46 (1997), 337-356.

3. P. Duren, Theory of $H^{p}$ Spaces, Academic Press, New York, 1970.

4. J. Garnett, Bounded analytic functions, Academic Press, New York, 1981.

5. Z. Hu, Extended Cesàro operators on mixed norm spaces, Proc. Amer. Math. Soc. 131 (2003), 2171-2179.

6. S. Krantz and S. Stević, On the iterated logarithmic Bloch space on the unit ball, Nonlin. Anal. TMA 71 (2009), 1772-1795.

7. J. Laitila, S. Miihkinen and P. Nieminen, Essential norms and weak compactness of integration operators, Arch. Mech. 97 (2011), 39-48.

8. P. Li, J. Liu and Z. Lou, Integral operators on analytic Morrey spaces, Sci. China 57 (2014), 1-15.

9. S. Li and S. Stević, Volterra type operators on Zygmund space, J. Inequal. Appl. 2007 (2007), 10 pages.

10. , Riemann-Stieltjes operators between different weighted Bergman spaces, Bull. Belg. Math. Soc. 15 (2008), 677-686.

11. , Cesàro type operators on some spaces of analytic functions on the unit ball, Appl. Math. Comp. 208 (2009), 378-388.

12. Integral-type operators from Bloch-type spaces to Zygmund-type spaces, Appl. Math. Comp. 215 (2009), 464-473.

13. J. Liu, Z. Lou and C. Xiong, Essential norms of integral operators on spaces of analytic functions, Nonlin. Anal. 75 (2012), 5145-5156.

14. C. Pommerenke, Schlichte Funktionen and analytische Funktionen vonbeschränkter mittlerer Oszillation, Comment. Math. Helv. 52 (1977), 591-602.

15. A. Siskakis and R. Zhao, A Volterra type operator on spaces of analytic functions, Contemp. Math. 232 (1999), 299-311.

16. S. Stević, On a new integral-type operator from the Bloch space to Bloch-type spaces on the unit ball, J. Math. Anal. Appl. 354 (2009), 426-434.

17. , On an integral-type operator from logarithmic Bloch-type and mixed-norm spaces to Bloch-type spaces, Nonlinear Anal. TMA 71 (2009), 63236342 .

18. M. Tjani, Distance of a Bloch function to the little Bloch space, Bull. Austral. Math. Soc. 74 (2006), 101-119.

19. R. Zhao, On a general family of function spaces, Ann Acad Sci. 105 (1996), 56 pages.

20. , New criteria of Carleson measure for Hardy spaces and their application, Complex Var. Ellip. Equat. 55 (2010), 633-646.

21. X. Zhu, Volterra composition operators on logarithmic Bloch spaces, Banach J. Math. Anal. 3 (2009), 122-130. 
22. Z. Zhuo and S. Ye, Volterra-type operators from analytic Morrey spaces to Bloch space, J. Integral Equations Appl. 27 (2015), 289-309.

School of Sciences, Anhui University of Science and Technology, Huainan, AnHui 232001, China

Email address: hope189@163.com

Department of Mathematics, Jiaying University, 514015, Meizhou, GuangDONG, CHINA

Email address: jyuzxl@163.com 